\title{
Chondroitin sulphate: unmasking auto-immune hepatitis
}

\author{
Mohamed Barakat ${ }^{1}$, Negar Niknam², Adina Valceanu ${ }^{1}$, Leelavathi Kasturi ${ }^{1}$ \\ ${ }^{1}$ Icahn School of Medicine at Mount Sinai - Queens Hospital Centre, NY, USA \\ ${ }^{2}$ Maimonades Medical Centre, NY, USA
}

Gastroenterology Rev 2019; 14 (4): 302-303

DOI: https://doi.org/10.5114/pg.2019.88173

\begin{abstract}
Address for correspondence: Mohamed Barakat MD, Icahn School of Medicine at Mount Sinai - Queens Hospital Centre, NY, USA, e-mail: mo_barakat@hotmail.com
\end{abstract}

Auto-immune hepatitis (AlH) is a chronic liver condition first described in the 1950s, which is characterised by the presence of immunological features. Triggers for auto-immune hepatitis are hypothesised to be environmental factors, viruses, drugs, and herbal supplements. Herein we present a case of auto-immune hepatitis unmasked by a chondroitin sulphate supplement.

A 64-year-old woman with persistent mildly elevated liver function tests (LFTs); aspartate aminotransferase (AST) 36-114 U/l, alanine aminotransferase (ALT) 24-86 U/I and minimally elevated anti-nuclear antibody (ANA) and anti-smooth muscle antibody (ASMA) titres (1:40), was found to have a sudden increase in her LFTs during her eighth year of follow-up. AST was $543 \mathrm{U} / \mathrm{l}, \mathrm{ALT} 650 \mathrm{U} / \mathrm{l}$, alkaline phosphatase $139 \mathrm{U} / \mathrm{l}$, total bilirubin $1.4 \mathrm{mg} / \mathrm{dl}$, and $\gamma$-glutamyl transferase $285 \mathrm{U} / \mathrm{l}$. One month prior to the office visit, the patient started taking a chondroitin supplement for bilateral knee osteoarthritis. After discontinuation of chondroitin sulphate, her LFTs normalised over the course of 4 months. Hepatitis serology, iron studies, and autoimmune hepatitis workup was repeated, which revealed negative viral hepatitis serology, normal iron studies, and an increase in her anti-nuclear antibody titre (ANA) $1: 2560$, anti-smooth muscle antibody (ASMA) titre 1 : 160, and negative AMA. Abdominal ultrasound revealed mild coarse echotexture of the liver. A liver biopsy was performed, which was suggestive of autoimmune hepatitis with moderate activity and cirrhosis (Figures 1, 2). The international AlH score was 7, which is consistent with a definite diagnosis of $\mathrm{AlH}$. The patient was started on azathioprine and prednisone for her newly established auto-immune hepatitis.

In our patient a history of mildly elevated LFTs and positive ANA and ASMA indicate that the patient had a history of mild, undiagnosed auto-immune hepatitis; however, the consumption of chondroitin sulphate unmasked her disease, as evident by the sudden increase in LFTs, ANA, and ASMA. Herbal preparations and supplements are often thought of as natural alternative remedies to medication without adverse effects [1]. Chondroitin sulphate is a sulphated glycosaminoglycan and has become a widely used dietary supplement for the treatment of osteoarthritis [2]. Although it is a generally well tolerated dietary supplement, chondroitin sulphate has been reported to cause drug-induced liver injury in select patients and in patients with chronic liver disease [3]. A chondroitin sulphate-induced liver injury mimicking auto-immune hepatitis was reported by Felden, in which the patient had negative immunologic markers; however, the condition was proven by liver biopsy [4]. Drug-induced auto-immune hepatitis is rare, and most cases are caused by minocycline and nitrofurantoin. The mechanism by which it causes hepatotoxicity and its relation to auto-immune hepatitis is not fully understood; however, hypotheses include metabolite-mediated immunoallergic reaction or drug-induced autoimmunity [5]. In contrast to our patient, autoimmune markers were elevated, suggesting autoimmune hepatitis rather than a drug-induced liver injury mimicking autoimmune hepatitis or a drug-induced autoimmune hepatitis.

Supplements containing chondroitin sulphate may not be suitable for patients with chronic liver disease and may be associated with auto-immune hepatitis. Further investigation is warranted.

\section{Conflict of interest}

The authors declare no conflict of interest. 


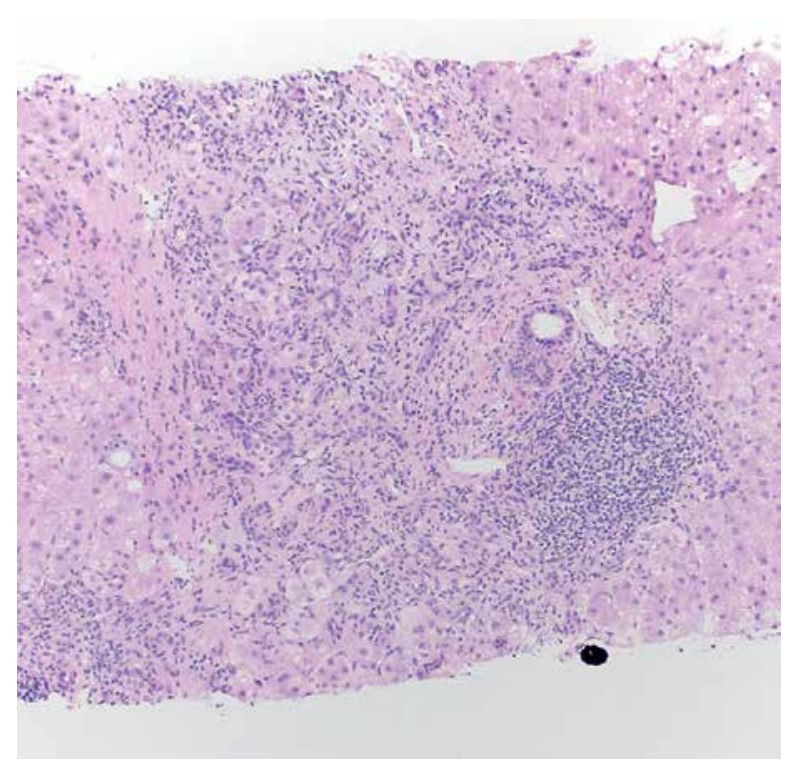

Figure 1. Portal triad with inflammation and interface hepatitis

\section{References}

1. Marignani M, Gallina S, Di Fonzo M, et al. Use and safety perception of herbal remedies in patients with liver/biliary tract disorders: an Italian study. J Clin Gastroenterol 2010; 44 Suppl 1: S54-7.

2. Vista ES, Lau CS. What about supplements for osteoarthritis? A critical and evidenced-based review. Int J Rheum Dis 2011; 14: 152-8.

3. Cerda C, Bruguera M, Parés A. Hepatotoxicity associated with glucosamine and chondroitin sulfate in patients with chronic liver disease. World J Gastroenterol 2013; 19: 5381-4.

4. von Felden J, Montani M, Kessebohm K, Stickel F. Drug-induced acute liver injury mimicking autoimmune hepatitis after intake of dietary supplements containing glucosamine and chondroitin sulfate. Int J Clin Pharmacol Ther 2013; 51: 219-23.

5. Czaja AJ. Drug-induced autoimmune-like hepatitis. Dig Dis Sci 2011; 56: 958-76.

Received: 10.12.2017

Accepted: 2.04 .2019

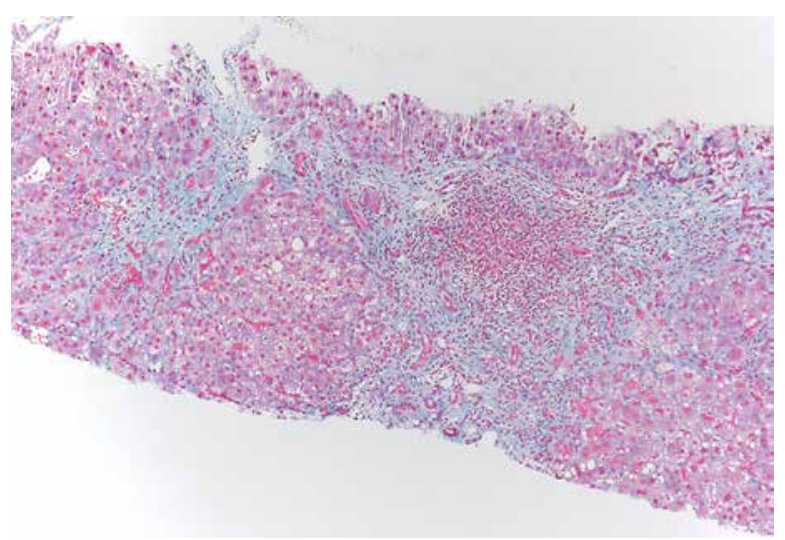

Figure 2. Trichrome stains showing bridging fibrosis with nodules 